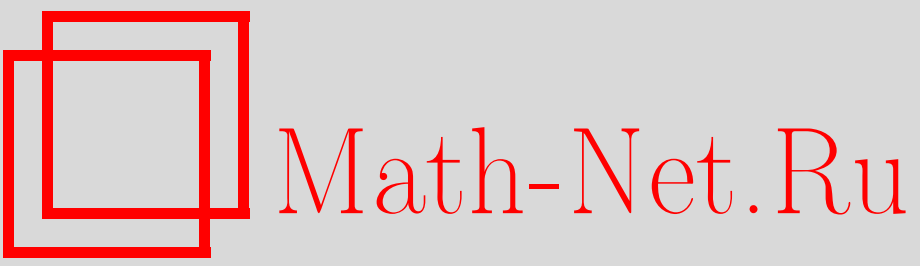

С. М. Натанзон, С. В. Шадрин, Топологическая классификация унитарных функций произвольного рода, УМН, 2000, том 55, выпуск 6, 151-152

DOI: https://doi.org/10.4213/rm350

Использование Общероссийского математического портала Math-Net.Ru подразумевает, что вы прочитали и согласны с пользовательским соглашением

http://www.mathnet.ru/rus/agreement

Параметры загрузки:

IP : 54.157 .27 .8

26 апреля 2023 г., 17:39:04 


\title{
ТОПОЛОГИЧЕСКАЯ КЛАССИФИКАЦИЯ УНИТАРНЫХ ФУНКЦИЙ ПРОИЗВОЛЬНОГО РОДА
}

\author{
С. М. НАТАНЗОН, С. В. ШАДРИН
}

1. Унитарной функцией мы называем тройку $(P, \tau, f)$, где $P$ - компактная риманова поверхность, $\tau: P \rightarrow P$ - антиголоморфная инволюция (т.е. $(P, \tau)$ - вещественная алгебраическая кривая) и $f: P \rightarrow \overline{\mathbb{C}}=\mathbb{C} \cup \infty$ - мероморфная функция, удовлетворяющая условию $f(\tau(z))=$ $(\overline{f(z)})^{-1}$. Унитарными являются, например, мономы $h_{n}(z)=z^{n}$ на вещественной кривой $(\overline{\mathbb{C}}, \eta)$, где $\eta(z)=\bar{z}^{-1}$. Унитарные функции $\left(P_{1}, \tau_{1}, f_{1}\right)$ и $\left(P_{2}, \tau_{2}, f_{2}\right)$ считаются совпадающими, если существует биголоморфное отображение $\varphi: P_{1} \rightarrow P_{2}$ такое, что $f_{1}=f_{2} \varphi$ и $\varphi \tau_{1}=\tau_{2} \varphi$. Естественная топология пространства мероморфных функций [1] наделяет множество унитарных функций структурой топологического пространства.

Статья посвящена топологической классификации унитарных функций с фиксированным топологическим типом дивизора полюсов. Соответствующие топологические инварианты аналогичны инвариантам вещественных мероморфных функций [1]-[3] и выделяют компоненту связности в пространстве унитарных функций. Кроме того, эти инварианты хорошо согласованы с мультипликативной структурой унитарных функций.

2. Множество вещественных точек вещественной алгебраической кривой $(P, \tau)$ совпадает с множеством $P^{\tau}$ неподвижных точек инволюции $\tau$. Оно состоит из простых попарно непересекающихся замкнутых контуров, называемых овалами. Если множество $P \backslash P^{\tau}$ несвязно (связно), то кривая называется разделяющей (неразделяющей). Топологическим типом кривой $(P, \tau)$ называется набор чисел $(g, k, \varepsilon)$, где $g$ - род поверхности $P, k$ - число овалов и $\varepsilon$ - число, равное 1 для разделяющих кривых и 0 для неразделяющих.

Топологическим типом дивизора полюсов мероморфной функции $f: P \rightarrow \overline{\mathbb{C}}$ называется набор чисел $A=\left(a_{1}, \ldots, a_{m}\right)$ такой, что $f^{-1}(\infty)=a_{1} p_{1}+\cdots+a_{m} p_{m}$, где $p_{i} \neq p_{j}$.

Рассмотрим мероморфную функцию $f: P \rightarrow \overline{\mathbb{C}}$ степени $n$ на кривой рода $g>0$ такую, что все ее критические значения, кроме 0 и $\infty$, простые. Существует и единственно [4] число $1 \leqslant r<n$, делящее $n$, такое, что группа монодромии $f$ порождается подстановками $(1, \ldots, n)$ и $(1, r+1)$, и $f$ представляется в виде композиции $h_{r} \circ f_{1}$ для некоторой мероморфной функции $f_{1}: P \rightarrow \overline{\mathbb{C}}$. Мы будем называть число $r$ характеристикой функции $f: P \rightarrow \overline{\mathbb{C}}$, полагая по определению $r=1$ для функций на сффере $\overline{\mathbb{C}}$.

3. Индексом унитарной функции $(P, \tau, f)$ на овале $c \subset P^{\tau}$ назьвается число обходов нуля точкой $f(p)$ при однократном обходе точкой $p$ овала $c$. Топологическим типом унитарной функции $(P, \tau, f)$ на неразделяющей вещественной кривой называется набор чисел $(g, n, 0|I| A, r)$, где $(g, k, 0)$ - топологический тип кривой $(P, \tau), n$ - число листов накрытия $f$, $I=\left(i_{1}, \ldots, i_{k}\right)$ - совокупность индексов функции $(P, \tau, f)$ на овалах, $A$ - топологический тип дивизора полюсов функции $f$ и $r$-характеристика функции $f^{*}: P \rightarrow \overline{\mathbb{C}}$, близкой к $f$, имеющей тот же топологический тип дивизоров полюсов и нулей и имеющей простые ненулевые конечные критические значения.

Обозначим через $H(g, n, 0|I| A, r)$ множество унитарных функций топологического типа $(g, n, 0|I| A, r)$.

Tеорема 1. Множество $H=H(g, n, 0|I| A, r)$ непусто, если и только если $1 \leqslant$ $r<n ; r=1$, если $g=0 ; r$ делит $n, I$ u $A ; n=\sum_{j=1}^{m} a_{j} ; 0 \leqslant k \leqslant g ; \sum_{j=1}^{k} i_{j} \leqslant$ $n-2$ и $\sum_{j=1}^{k} i_{j} \equiv n(\bmod 2)$. В этом случае $H$ - связное пространство размерности $2(g+m-1)$.

4. Индексам унитарной функции $(P, \tau, f)$ на овалах разделяющей кривой $(P, \tau)$ можно приписать знаки. А именно, ориентируем $\overline{\mathbb{C}}$ и $P$ так, чтобы $f$ сохраняла ориентацию. Пусть $P_{1}$ и $P_{2}$

Работа первого автора выполнена при частичной финансовой поддержке Российского фонда фундаментальных исследований (грант № 98-01-00612). 
- компоненты связности дополнения $P \backslash P^{\tau}$. Ориентация поверхности $P_{1}$ и диска $D=\{z \in \overline{\mathbb{C}} \mid$ $|z| \leqslant 1\}$ индуцирует ориентацию на их границах $P^{\tau}=\partial P_{1}$ и $\partial D$. Ввиду $f\left(P^{\tau}\right) \subset \partial D$ эта ориентация позволяет определить степени $\left(i_{1}, \ldots, i_{k}\right)$ функции $(P, \tau, f)$ на овалах кривой $(P, \tau)$. Набор степеней определен с точностью до замены $\left(i_{1}, \ldots, i_{k}\right) \mapsto\left(-i_{1}, \ldots,-i_{k}\right)$, связанной с произволом в выборе компоненты связности дополнения $P \backslash P^{\tau}$. Модули степеней совпадают с соответствующими индексами функции $(P, \tau, f)$ на овалах.

Топологическим типом унитарной функции $(P, \tau, f)$ на разделяющей вещественной кривой назьвается набор чисел $\left(g, n, 1|I| A_{1}, A_{2}, r\right)$, где $(g, k, 1)$ - топологический тип кривой $(P, \tau), n$ - число листов накрытия $f, I$ - совокупность степеней функции $(P, \tau, f)$ на овалах, $A_{i}=\left(a_{1}^{i}, \ldots, a_{m_{i}}^{i}\right)$ - топологический тип дивизора полюсов ограничения функции $f$ на $P_{i}$ и $r$ - характеристика функции $f^{*}: P \rightarrow \overline{\mathbb{C}}$, близкой к $f$, имеющей тот же топологический тип дивизоров полюсов и нулей и имеющей простые ненулевые конечные критические значения. Говорят, что топологический тип допускает расширение, если $\left|\sum_{j=1}^{k} i_{j}\right|<\sum_{j=1}^{k}\left|i_{j}\right|=n-2 r$.

Обозначим через $H\left(g, n, 1|I| A_{1}, A_{2}, r\right)$ множество унитарных функций топологического типа $\left(g, n, 1|I| A_{1}, A_{2}, r\right)$. Очевидно, что $H\left(g, n, 1|I| A_{1}, A_{2}, r\right)=H\left(g, n, 1|-I| A_{2}, A_{1}, r\right)$.

Теорема 2. Множество $H=H\left(g, n, 1|I| A_{1}, A_{2}, r\right)$ непусто, если и только если $1 \leqslant r<n ; \quad r=1, \quad$ если $g=0 ; \quad r$ делит $n, \quad I, \quad A_{1} \quad u \quad A_{2} ; \quad 1 \leqslant k \leqslant g+1$; $k \equiv g+1(\bmod 2) ; \quad \sum_{j=1}^{k} i_{j} \equiv n(\bmod 2) ; \quad \sum_{j=1}^{m_{1}} a_{j}^{1}=\sum_{j: i_{j}>0} i_{j}+\left(n-\sum_{j=1}^{k}\left|i_{j}\right|\right) / 2 ;$ $\sum_{j=1}^{m_{2}} a_{j}^{2}=-\sum_{j: i_{j}<0} i_{j}+\left(n-\sum_{j=1}^{k}\left|i_{j}\right|\right) / 2$ u либо $n=1, g=0, i_{1}= \pm 1$, либо $n=2$, $k=g+1, i_{1}=\cdots=i_{k}=0$, либо $n \geqslant 2,\left|\sum_{j=1}^{k} i_{j}\right|=n, i_{j} \neq 0$, либо $n \geqslant 3, \sum_{j=1}^{k}\left|i_{j}\right| \leqslant n-2$. Кроме того, если множество $H$ непусто и топологический тип $\left(g, n, 1|I| A_{1}, A_{2}, r\right)$ не допускает расширения, то $H$ - связное пространство размерности $2\left(g+m_{1}+m_{2}-1\right)$.

5. Пусть теперь топологический тип $\left(g, n, 1|I| A_{1}, A_{2}, r\right)$ допускает расширение и множество $H=H\left(g, n, 1|I| A_{1}, A_{2}, r\right)$ непусто. Для каждой $(P, \tau, f) \in H$ рассмотрим компоненту связности $H_{0}$ пространства $H$, содержащую функцию $(P, \tau, f)$. Тогда найдется функция $\left(P^{\prime}, \tau^{\prime}, f^{\prime}\right) \in H_{0}$ такая, что $P_{1}^{\prime} \cap f^{\prime-1}(D)$ - связная поверхность (здесь $P_{1}^{\prime}$ - та из связных компонент дополнения $P^{\prime} \backslash\left(P^{\prime}\right) \tau^{\prime}$, с помощью которой определяется топологический тип $\left.\left(g, n, 1|I| A_{1}, A_{2}, r\right)\right)$. Рacuuренным топологическим типом функции $(P, \tau, f) \in H$ называется набор чисел $(g, n, 1 \mid I$, $\left.\chi \mid A_{1}, A_{2}, r\right)$, где $\chi$ - род поверхности $P_{1}^{\prime} \cap f^{\prime-1}(D)$. Можно доказать, что расширенный топологический тип определен корректно с точностью до замены $\left(g, n, 1|I, \chi| A_{1}, A_{2}, r\right) \mapsto(g, n, 1 \mid-I$, $\left.(g-k+1) / 2-\chi \mid A_{2}, A_{1}, r\right)$.

Обозначим через $H\left(g, n, 1|I, \chi| A_{1}, A_{2}, r\right)$ подмножество $H$, состоящее из всех функций расширенного топологического типа $\left(g, n, 1|I, \chi| A_{1}, A_{2}, r\right)$. Очевидно, что $H\left(g, n, 1|I, \chi| A_{1}, A_{2}, r\right)$ $=H\left(g, n, 1|-I,(g-k+1) / 2-\chi| A_{2}, A_{1}, r\right)$.

Tеорема 3. Множество $H_{0}=H\left(g, n, 1|I, \chi| A_{1}, A_{2}, r\right)$ непусто, если и только если $0 \leqslant \chi \leqslant(g-k+1) / 2$. В этом случае $H_{0}$ - связное пространство размерности $2\left(g+m_{1}+m_{2}-1\right)$.

6. Доказательства теорем строятся по той же схеме, что и в [3], но с использованием теоремы [4] о топологической классификации разветвленных накрытий над сферой с двумя непростыми критическими значениями.

\section{СПИСОК ЛИТЕРАТУРЫ}

[1] Натанзон С. М. // Докл. АН СССР. 1984. Т. 279. № 4. С. 803-805. [2] Натанзон С. М. // Докл. АН СССР. 1987. Т. 297. № 1. C. 40-43. [3] Natanzon S. M. // Selecta Math. Soviet. 1993. V. 12. № 3. P. 251-291. [4] Wajnryb B. // Indag. Math. 1996. V. 7. P. 549-558.

Московский государственный университет им. М. В. Ломоносова; Независимый московский университет

E-mail: natanzon@mccme.ru, shadrin@mccme.ru
Принято редколлегией 03.10 .2000 